\title{
Pendulum dynamics in the amusement park
}

\author{
Pol Gurri ${ }^{1}$, David Amat ${ }^{1}$, Joan Espar ${ }^{1}$, Jordi Puig ${ }^{1}$, Gerard \\ Jimenez $^{1}$, Lluc Sendra ${ }^{1}$ and Luis C. Pardo ${ }^{2}$ \\ ${ }^{1}$ Universitat Politècnica de Catalunya, Campus Nord UPC 08034 Barcelona, Spain \\ ${ }^{2}$ Grup de Caracterització de Materials, Departament de Física, EEBE, Universitat \\ Politècnica de Catalunya, Eduard Maristany 19, 08930, Barcelona, Catalonia, Spain \\ E-mail: polgurri@gmail.com
}

\begin{abstract}
Pendulums have long been used to to determine the kinematics of noninertial rotating systems. A proper study of this classical mechanical device under the action of a rotating reference frame can fully describe the behavior of such non-inertial systems. In such venue, we have placed a pendulum inside a rotating plane ride in an amusement park, and determined the parameters defining the rotation of the ride by solely analyzing the pendulum's motion. We present two different methodologies to determine the motion patterns of the ride as well as a computer-based reproduction of the trajectory of the pendulum throughout the ride.
\end{abstract}

PACS numbers: $00.00,20.00,42.10$

Keywords: Article preparation, IOP journals Submitted to: J. Phys. A: Math. Gen.

\section{Introduction}

Amusement parks offer an unique opportunity to perform experiments in environments hard to reproduce in a normal laboratory. For this reason the project FISIDABO offers to the students the opportunity to bring the experiments that have been designed and built by them to Tibidabo, the amusement park of Barcelona. The aim of this motivational activity is twofold. First of all, the amusement park offers to the students the possibility to do the experiments when the park is closed to the public. This allows them to perform the experiment and obtain the data in a controlled environment that can be tuned by the technicians of the park to achieve the best results. Second, once the experiment is performed and the data is analyzed, the experiment can be used on its own to show other students the physic principles on which the performed experiment is based. The goal of the project presented in this paper is to show a project developed by students of the Physics Engineering degree at the "Universitat Politècnica de Catalunya", and show how it can be used to study the dynamics in non-inertial frames in any other ride that spins like the "red plane" of Tibidabo (see Fig. 1). 
Dynamics can be as complicated as the system under study. However, even simple dynamical problems, as an oscillating pendulum, can be converted into very complicated ones when the frame where the motion takes place is not inertial. In addition, performing experiments in non-inertial frames is a difficult task that cannot be achieved in normal laboratories that are fixed to the ground, as the ones we can find in university buildings. One of the most iconic rides in Tibidabo is a plane that hangs from a structure anchored to a spinning platform. The plane, thus, seems to fly in circles and therefore constitutes a well suited non-inertial frame where dynamics experiments in a non-inertial frame can be performed. We have chosen the "simple" motion of a pendulum like the one that used Foucault to prove that the earth rotates around an axis. Our aim is, indeed, the same as that of Foucault: to prove that we can obtain, from the kinematics of a pendulum, information about the movement of the rotating frame in which the pendulum is embeded. We would like to stress the fact that both the experiment and the analysis can be done in any other similar rotating frame and is not reduced to the particularities of the Tibidabo ride.

The Foucault pendulum is a well-renowned object in the world of physics as it was one of the first non-astronomical experiments to demonstrate the rotation of the Earth. In 1851, the French physicist Léon Foucault suspended a $28 \mathrm{Kg}$ mass with a 67 meterslong wire from the top of the dome of the Panthéon in Paris. A pendulum at rest in an inertial frame will oscillate continually in a single plane, but for a pendulum positioned on a rotating system, the swinging motion will also rotate with respect the reference system. Because of the rotation of the Earth, the resulting Foucault's pendulum plane of oscillation appeared to rotate at a rate of 11 degrees per hour, taking $~ 32.7$ hours to accomplish a complete revolution. Although the Foucault pendulum was originally used for astronomical purposes, it can also be exploited in rotating systems to derive the parameters that define their intrinsic motion [1].

Far from trying to reproduce Foucault's experiment we study a pendulum inside a rotating frame to fully describe its motion, identify the main properties of the system's trajectory and, ultimately, obtain the radius of rotation $(R)$ of the Tibidabo's red plane. To do so, we placed a pendulum inside the Tibidabo's plane and analyzed its movement. The system that we aimed to parametrize consisted thus on a plane that orbits around a fixed point and a pendulum hanged from the roof of the plane, as exemplified in Figure 1 .

We present two different methodologies to constraint the radius of rotation of the ride, which are described in section 2 and 3. While section 2 provides an in-situ, orderof-magnitude measurement for such radius, section 3 describes a more precise procedure that yielded far more accurate results. Section 4 exhibits a basic theoretical study of the dynamics of the pendulum which combined with the previous results and some computer based procedures was able to reproduce the $3 \mathrm{D}$ trajectory of the pendulum inside the ride. We briefly discuss our results and conclude in section 5 . 


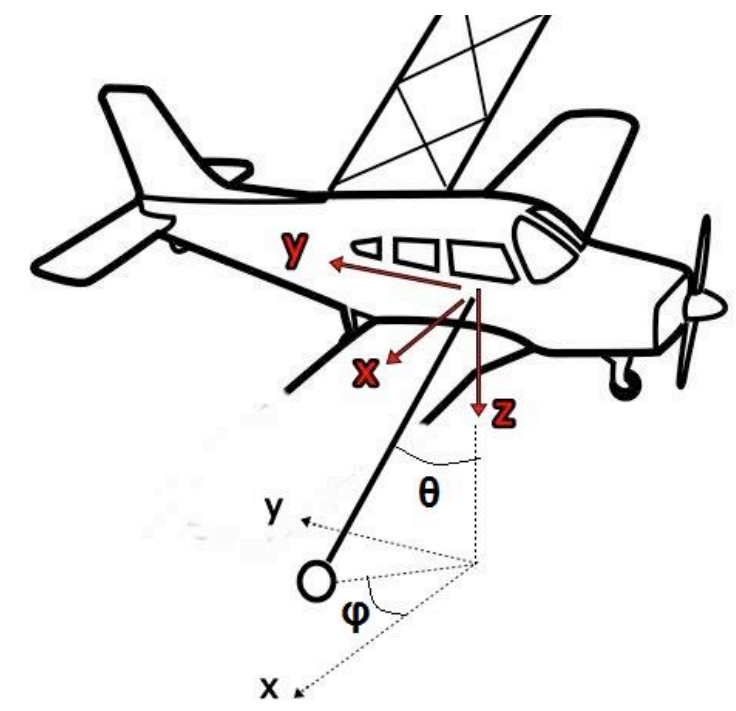

Figure 1. Conceptual representation of the Tibidabo's plane. The scheme includes the Cartesian (red bold letters) and Spherical (black letters) axis used in this work. The origin of both reference systems is located at the center of the plane's roof, where the pendulum is attached.

\section{A simple approach: calculating the radius and period}

This section describes a fairly simple methodology that we used to obtain a first approximation of the value of the radius of ration of the Tibidabo's plane. Such measurements were not intended to be precise but to provide an order-of-magnitude analysis. The key concept behind this study is that the pendulum swings in a noninertial, rotating frame that inflicts dynamical effects on the pendulum. Therefore, the pendulum's oscillation plane seems to rotate when observed within the system. These effects in the non-inertial frame of the plane include two forces: a force in the direction of the normal acceleration (outwards in the direction of the radius) and a fictitious force that makes the pendulum rotate (if seen from the inside of the plane). We are particularly interested in the former one as it moves the resting position of the pendulum and separates it from a vertical position as shown in Figure 2. The displacement length from the vertical is proportional to the normal acceleration of the system and therefore to the speed and radius of rotation of the plane. Figure 2 illustrates a schematic representation of the pendulum under the effects of the planes rotation. We use $g$ for gravity, $X$ for the displacement from the vertical and $a_{c}$ for the acceleration responsible of such displacement.

We set the pendulum at rest into the equilibrium position $(\theta=0)$ when the plane was not moving. Once the the plane was spinning, we measured the displacement from the vertical $(\theta=0)$ using a measuring-tape, which led to a result of $\simeq 0.10 \pm 0.02 \mathrm{~m}$. Knowing the length of the pendulum $(1.8 \mathrm{~m})$, we used simple trigonometry to obtain $\theta$, the angle between the resting position and the vertical (see Figure 2). This leads to the 
following relation:

$$
\theta=\arcsin \left(\frac{X}{l}\right)=\arcsin \left(\frac{0.10 \pm 0.02}{1.8}\right) \simeq 0.06 \pm 0.01 \mathrm{rad}
$$

In the resting position the sum of all forces must be null, therefore, by using Newton's second law, it is possible to obtain the magnitude of the normal acceleration, $a_{c}$, which is responsible for the displacement $X$. Equation 2 imposes equilibrium of forces at the resting position, and later provides the value of the normal acceleration in our scenario.

$$
m a_{c} \cos (\theta)=m g \sin (\theta) \rightarrow a_{c}=g \cdot \tan (\theta)=g \cdot \tan (0.06 \pm 0.01) \simeq 0.5 \pm 0.1 \mathrm{~ms}^{-2}(2)
$$

Once the acceleration was no longer unknown, we proceeded to resolve the period of rotation of the plane. To do so, we let the pendulum oscillate and measured with a timer how long it took for the pendulum to return to a certain initial position. With a period of $T \simeq 35 \pm 1 \mathrm{~s}$ and knowing the acceleration, we used Equation 3 to make a rough approximation of the radius of rotation.

$$
R=a_{c}\left(\frac{T}{2 \pi}\right)^{2}=0.5 \pm 0.1\left(\frac{35 \pm 1}{2 \pi}\right)^{2} \simeq 17 \pm 4 \mathrm{~m}
$$

This methodology provided a first approach to the radius of $R \simeq 17 \pm 4 \mathrm{~m}$. The actual length of the radius was of $20 \mathrm{~m}$, which means that our measurement is in the right order of magnitude but lacks plenty of accuracy, mostly due to the imprecise measurement of the displacement from the vertical with the measuring-tape.

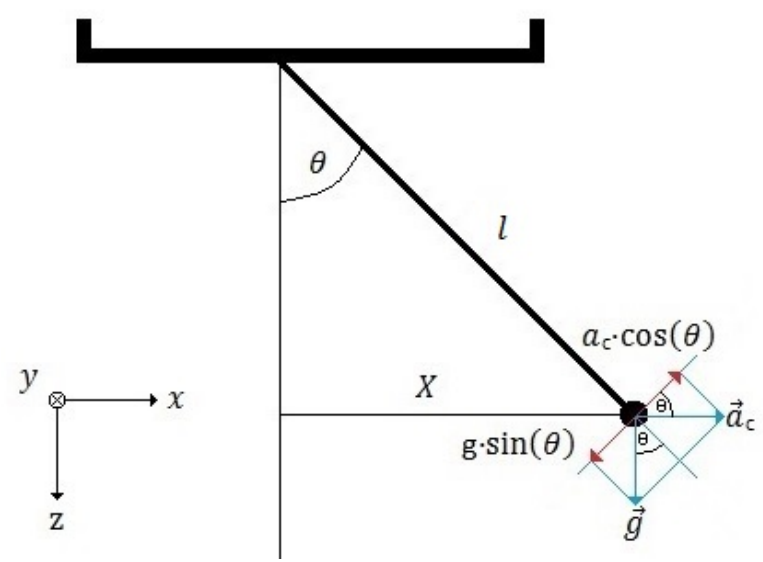

Figure 2. Simple scheme of a pendulum inside a non-inertial rotating system. All accelerations are drawn as well as their division in an alternative system of reference induced by the direction of the thread sustaining the pendulum. 


\section{A sophisticated appraoch: A computer-based analysis}

This section details a different and more precise approach to obtain the radius of rotation of the red plane ride in Tibidabo. Instead of relying on "in-situ" measurements, we recorded the movement of the pendulum and later analyzed its behavior to come up with a more accurate value of the radius. We set a video camera in the plane's floor, just beneath the pendulum ( $\sim 40 \mathrm{~cm}$ from the bob's resting position), which was recording during whole experiment. Thus, we obtained a video-record containing the coordinates of the pendulum as a function of time within the plane.

The video file was later analyzed with the public domain, Java-based image processing program IMAGEJ, which allowed to obtain the $x_{\text {video }}$ and $y_{\text {video }}$ coordinates of the position of the pendulum's bob for each frame of the video (i.e. 30 measurements per second). We placed a ruler at the height of the pendulum's bob ( $\sim 40 \mathrm{~cm}$ from the camera) and recorded it with the same camera to relate distances in the video with real life lengths, and therefore, be able to extrapolate $x_{\text {video }}$ and $y_{\text {video }}$ to meters $(x$ and $y$ ). After analyzing every frame of the video and transforming the coordinates into meters, we obtained a list of $x$ and $y$ values over time.

Figure 3 displays the $x$ component of the pendulum over time. The shape of the function most resembles a double sinusoidal function $(\sin (a) \times \sin (b))$ being one $\sin$ due the plane rotation and another due the pendulum oscillation. Figure 3 also exhibits that the double sinusoidal is displaced from the origin (i.e. it has not a null mean), which is caused by the rotation of the plane. The mean of the function is analogous to the $X$ displacement introduced in Figure 2. We computed the mean of the signal in Figure 3 and plotted in red its value, which equals to $\langle x(t)\rangle \simeq X=0.114 \pm 0.005 \mathrm{~m}$.

To follow a similar procedure to that of Section 2 we also needed to know the period of rotation of the ride. To do so, we performed a spectral analysis of the data set, which, measuring the spectral density, characterizes the frequency content of a signal. Spectral analysis provides the main frequencies of a signal [2], which in our case are the rotation frequency of the plane and the oscillation frequency of the pendulum. Because the data set contains information about two correlated frequencies (a product of two sinusoidal functions) the spectral analysis results in two peaks at at $F 1=\left|f_{1}-f_{2}\right|$ and $F 2=f_{1}+f_{2}[2]$. The power spectral analysis of the data exhibited two clear peaks at $0.4286 \pm 0.0001 \mathrm{~Hz}$ and $0.4857 \pm 0.0001 \mathrm{~Hz}$, which set the two different frequencies to be $f_{1}=0.0285 \pm 0.0001 \mathrm{~Hz}$ and $f_{2}=0.4572 \pm 0.0001 \mathrm{~Hz}$. Frequencies can be transformed into periods with the relation $T=f^{-1}$, which provided a period for the plane ride of $T_{1}=T=35.1 \pm 0.1 \mathrm{~s}$ and $T_{2}=2.1875 \pm 0.0003 \mathrm{~s}$ for the pendulum's one. With $X$ and $T$ determined, we computed the value of the radius using once more equations 1 to 3 , and obtained an improved measurement for the radius of the ride of $R=19.5 \pm 0.9 \mathrm{~m}$. 


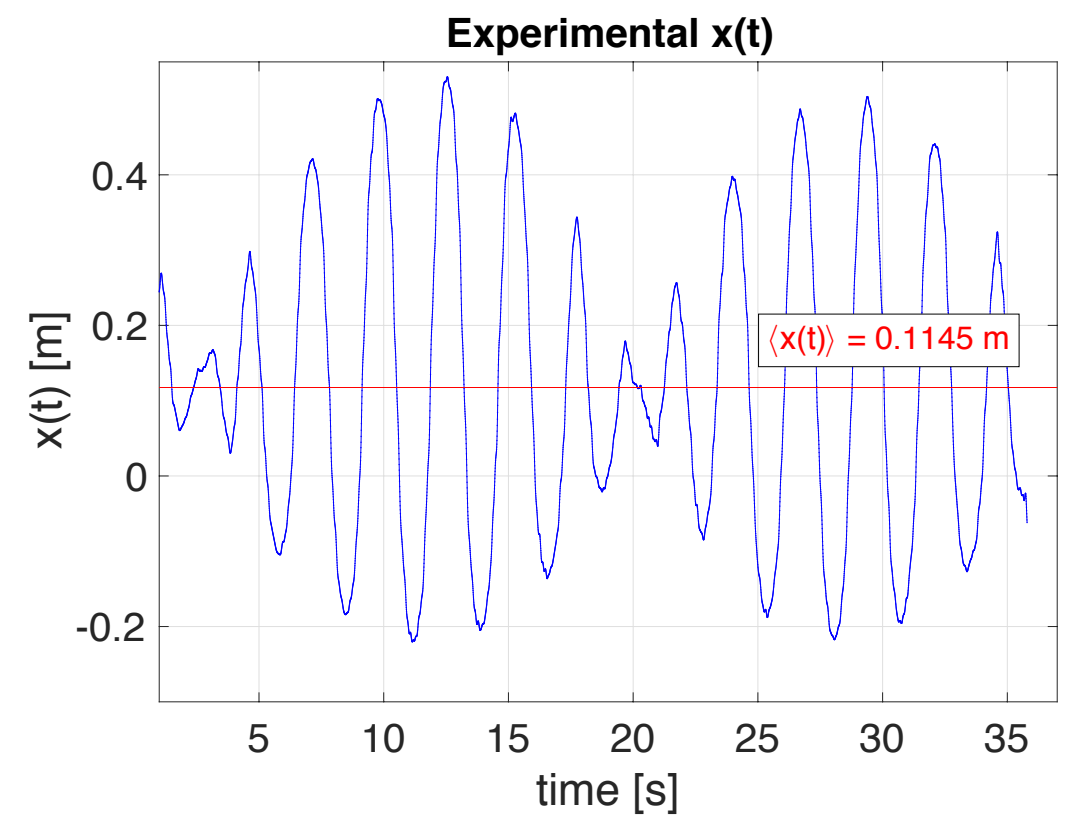

Figure 3. In blue, $x$ component of the trajectory of the pendulum over time obtained via video tracking. In red, the mean of the signal $\langle x(t)\rangle$, which also equals to $X=0.1145 \pm 0.005 \mathrm{~m}$.

\section{Determining trajectory}

Going beyond the constraining of the values that define the motion of the system we aimed to visualize a computer-based reconstruction of the trajectory of the pendulum inside the ride. This section provides an insight into the basic equations describing the motion of a Foucault pendulum with the aim of achieving a theoretical model of the trajectory of the pendulum inside the red plane. For a deep and exhaustive understanding of the following equations we point the readers to books specifically devoted to classical dynamics (e.g. [3]).

In order to easily describe the system, we designed two different reference frames, Cartesians to describe the motion of the plane and Sphericals for the pendulum (black and red respectively in Figure 1). Table 1 includes a legend with all the variables and symbols used in this work together with their units.

There are many approaches to formulate the equations governing the trajectory of the pendulum inside the ride. In this work we rely on Lagrangian dynamics, which provides a way of retrieving the trajectory of objects by solving the Euler-Lagrange equations. A Lagrangian is a particular function that contains all the information about the dynamics of the system. In our system, the Lagrangian can be defined as the potential energy of the system subtracted to its kinetic energy. The system we are trying to model consists of the pendulum and the ride together, and therefore the Lagrangian must consider energy contributions from both of them. Equation 4 presents the Lagrangian describing the dynamics of our system. Note that any variable with a dot in top of it represents the temporal derivative of this variable, and a double dot 
Table 1. Legend of all symbols, variables and units that appear in the theoretical analysis of the Foucault pendulum. All values have their usual symbols and a detailed explanation of them can be found in many theoretical books (e.g. [3]).

\begin{tabular}{lrr} 
Symbol & Variable & Units \\
\hline $\mathcal{L}$ & Lagrangian & {$[\mathrm{J}]$} \\
$\mathcal{H}$ & Hamiltonian & {$[\mathrm{J}]$} \\
$\mathcal{T}$ & Kinetic Energy & {$[\mathrm{J}]$} \\
$\mathcal{V}$ & Potential Energy & {$[\mathrm{J}]$} \\
$\mathrm{m}$ & Mass of Pendulum & {$[\mathrm{kg}]$} \\
$\mathrm{l}$ & Length of Pendulum & {$[\mathrm{m}]$} \\
$\phi$ & Second Spherical coordinate & {$[\mathrm{rad}]$} \\
$\theta$ & Third Spherical coordinate & {$[\mathrm{rad}]$} \\
$\omega$ & Angular Velocity & {$\left[\mathrm{rad} \cdot \mathrm{s}^{-1}\right]$} \\
$\mathrm{R}$ & Radius of Plane Rotation & {$[\mathrm{m}]$} \\
$\mathrm{g}$ & Gravity & {$\left[\mathrm{m} \cdot \mathrm{s}^{-2}\right]$} \\
$\mathrm{f}$ & Rotation Frequency & {$\left[\mathrm{s}^{-1}\right]$} \\
$\mathrm{T}$ & Rotation Period & {$[\mathrm{s}]$}
\end{tabular}

represents its second temporal derivative.

$$
\mathcal{L}=\frac{1}{2} m l^{2}\left(\dot{\theta}+\dot{\phi} \sin ^{2} \theta\right)+m g l \cos \theta+m w l^{2} \dot{\phi} \sin ^{2} \theta+m w^{2} l R \sin \theta \cos \phi+\frac{1}{2} m w^{2} l^{2} \sin ^{2} \theta(4)
$$

In Lagrangian mechanics, because of Hamilton's principle of stationary action, the dynamical evolution of a physical system is described by the solutions to the EulerLagrange equation. The Euler-Lagrange equation provides therefore, an advantageous way of obtaining the motion equations ruling a system given its Lagrangian. In our case, because the pendulum is described in Spherical coordinates with a fixed radius (pendulum length $l$ is always the same), the variables needed to fully describe the pendulum trajectory are $\theta$ and $\phi$. Due to the need of tracking two variables throughout time, the Euler-Lagrange equation translates into two different equations. Equation 5 and Equation 6 describe the evolution of $\theta$ and $\phi$ over time, and therefore, the pendulum's trajectory.

$$
\begin{aligned}
& \ddot{\theta}=(\dot{\phi}-w)^{2} \sin \theta \cos \theta-\frac{g}{l} \sin \theta+\frac{w^{2} R}{l} \cos \theta \cos \phi \\
& \ddot{\phi}=-\frac{w^{2} R}{l} \frac{\sin \phi}{\sin \theta}-2 \dot{\theta}(\dot{\phi}-w) \frac{\cos \theta}{\sin \theta}
\end{aligned}
$$

Equation 5 and Equation 6 have no analytic solution. Hence, in order to obtain the specific values that $\theta$ and $\phi$ take along time, we need to numerically integrate them. A common approach is to use a Runge-Kutta integrator of order five [4], which leads to something similar to having the functions $\theta(t)$ and $\phi(t)$. In order to numerically integrate equations 5 and 6 it is necessary to specify the initial conditions of the pendulum, which 
include the initial position in terms of $\theta$ and $\phi$ and initial speed in terms of $\dot{\theta}$ and $\dot{\phi}$. Also, to reproduce the trajectory of the pendulum inside the red plane one needs to know the specific properties of the system such as $R, l$ and $w=2 \pi / T$.

Using the values obtained in section 3 (i.e. $R \simeq 20 \mathrm{~m}, l=1.8 \mathrm{~m}$ and $T \simeq 35 \mathrm{~s}$ ) we were able to obtain the theoretical trajectory of the pendulum inside the red plane. In Figure 4 we plot a direct comparison between the theoretical trajectory and the experimental data. Left panels show the theoretical predictions, while right panels the experimental data points. In Figure 4 it is easy to appreciate a clear correlation between theory and experimental data, meaning that the theoretical model properly fits the observed data.

Furthermore, this methodology allowed us to also plot a 3D theoretical representation of the pendulum behavior within the plane, plotted in Figure 5 (left panel). We used a color scale for height to ease the understanding, being more pink as the trajectory gains height and bluer in its lower stages. To highlight the effects of the plane rotation on the pendulum we recreated the theoretical trajectory of the pendulum observed from the direction of movement of the plane (always facing the back of the plane). Figure 5 (right panel) shows the plane $x-z$ perspective, giving an idea of how the pendulum reaches different heights due to the acceleration induced by the plane.

\section{Conclusions}

We have seen how the proper study of a pendulum inside a non-inertial system can be sufficient to determine the intrinsic parameters ruling its motion. A proper model and some basic calculations and measurements were enough to determine the radius of rotation, the angular velocity and the period of the system and to prove that it is possible to obtain a $3 \mathrm{D}$ recreation of the trajectory of a pendulum inside a rotating system. Furthermore, this methodology can be implemented in systems where information from outside is not reachable as we treated the plane as a black box from which we could not get information of the exterior. We have also shown how such experiment can be performed in bizarre places, and how an amusement park could be used as an in-field, physics lab.

\section{Acknowledgments}

We thank the referees for all their valuable input.

This work would have not been possible without the Fisidabo project and all of its members and associations supporting to the project. We like to thank the amusement park Tibidabo for offering their facilities and believing in the project and the potential of young people. We also want to extend our heartfelt thanks to the professors Alvaro Messeguer and Francisco Marquès for their advice and help with the data treatment and theoretical model. And finally, we want to acknowledge the continuous efforts offered by Enginyeria Física from Universitat Politècnica de Catalunya. 

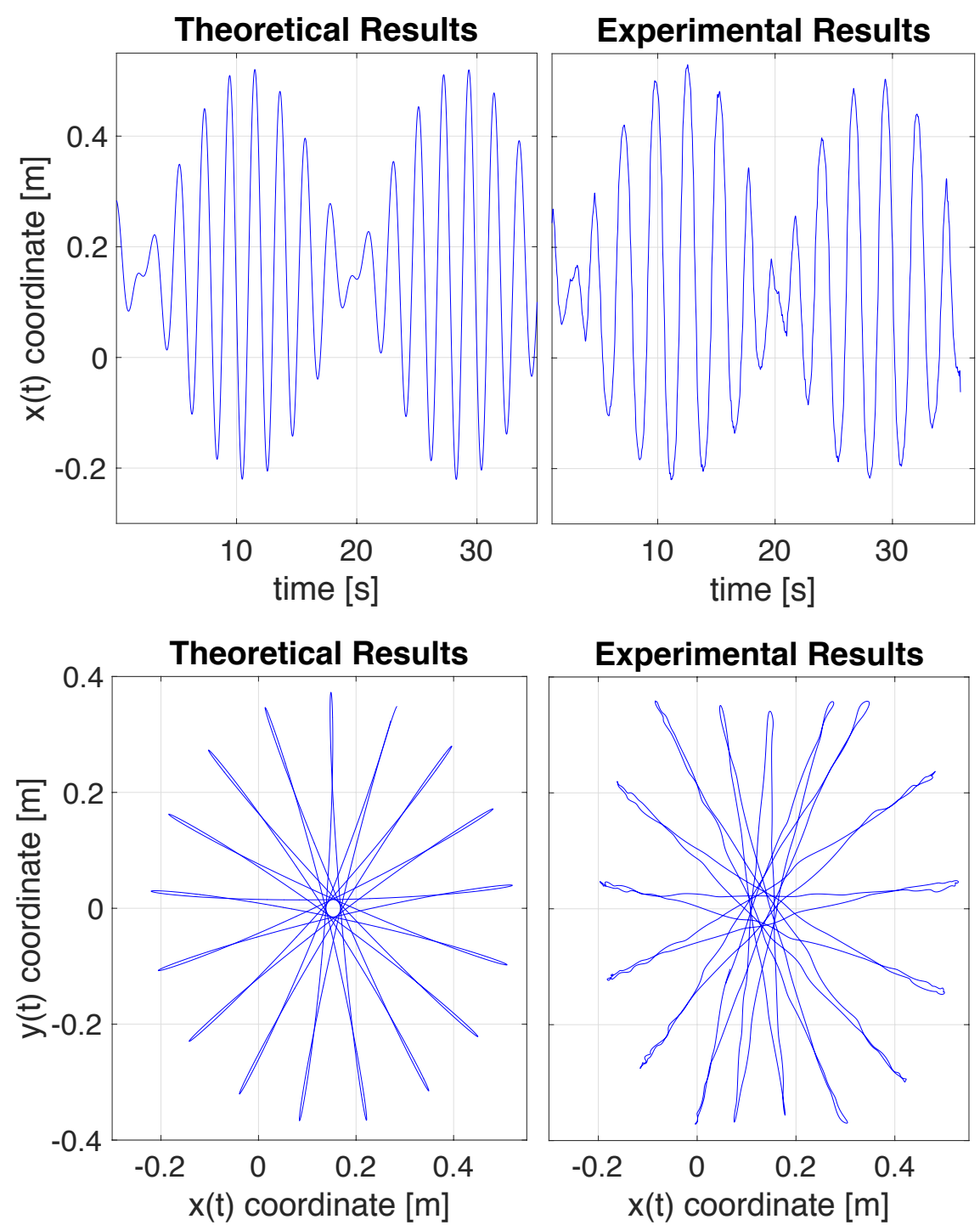

Figure 4. Direct comparison between the behavior predicted by theory and the actual data. Both right and left subfigures present on the left, theory predicted motion and at the right the video processed data of the trajectory. The top figure plots the value of $x$ coordinate over time while the bottom plot displays the $x$ and $y$ coordinates during an entire plane rotation.

\section{References}

[1] W. B. Somerville, The Description of Foucault's Pendulum, Q. J. R. Astron. Soc., (1972) 13,40-62

[2] Percival, Donald B., Walden, Andrew T., Spectral Analysis for Physical Applications, Cambridge University Press, Cambridge 1992.

[3] Goldstein, H. and Ferrer, J.F., Mecánica clásica, 1st edition Reverte, Barcelona 1987

[4] Atkinson, K., An Introduction to Numerical Analysis, 2nd edition Wiley, Minnesota, 1989 

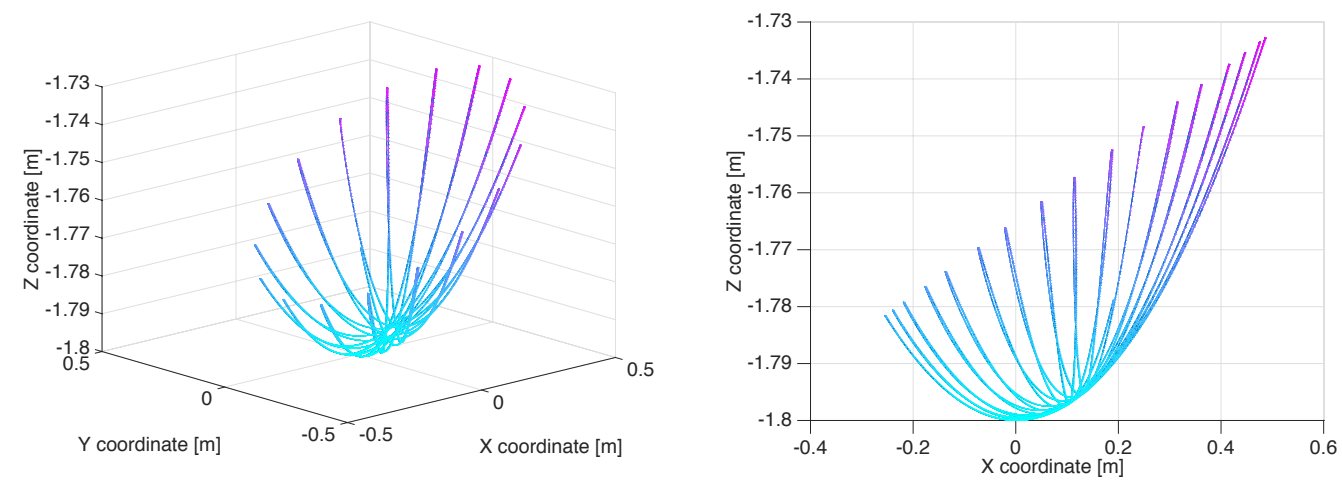

Figure 5. Theoretical representation of the 3D trajectory followed by the pendulum according to the obtained frequency values. The color gradient indicates height, being purple the greatest height and light blue the lowest elevation. 